\title{
Molecular Analyses of the Metallothionein Gene Family in Rice (Oryza sativa L.)
}

\author{
Gongke Zhoü, Yufeng $\mathrm{Xu}^{\#}$, Ji $\mathrm{Li}^{\#}$, Lingyan Yang and Jin-Yuan Liu* \\ Laboratory of Molecular Biology and Protein Science Laboratory of the Ministry of Education, \\ Department of Biological Sciences and Biotechnology, Tsinghua University, Beijing 100084, China
}

Received 16 April 2006, Accepted 7 June 2006

\begin{abstract}
Metallothioneins are a group of low molecular mass and cysteine-rich metal-binding proteins, ubiquitously found in most living organisms. They play an important role in maintaining intracellular metal homeostasis, eliminating metal toxification and protecting against intracellular oxidative damages. Analysis of complete rice genome sequences revealed eleven genes encoding putative metallothionein (OsMT), indicating that OsMTs constitute a small gene family in rice. Expression profiling revealed that each member of the OsMT gene family differs not only in sequence but also in their tissue expression patterns, suggesting that these isoforms may have different functions they perform in specific tissues. On the basis of OsMT structural and phylogenetic analysis, the OsMT family was classified as two classes and class I was subdivided into four types. Additionally, in this paper we also present a complete overview of this family, describing the gene structure, genome localization, upstream regulatory element, and exon/intron organization of each member in order to provide valuable insight into this OsMT gene family.
\end{abstract}

Keywords: Expression patterns, Gene family, Molecular characterization, Phylogenetic relationship, Rice metallothionein

\section{Introduction}

Metallothioneins (MTs) constitute a superfamily of evolutionally conserved, low molecular mass, cysteine-rich proteins that can bind metals via the thiol groups of their cysteine (Cys) residues (Coyle et al., 2002). Since being first purified as Cdbinding protein from horse kidney in 1956 (Vallee, 1991),

\footnotetext{
\#These authors contributed equally to this work.

* To whom correspondence should be addressed.

Tel: 86-10-62783845; Fax: 86-10-62772243

E-mail: liujy@mail.tsinghua.edu.cn
}

MTs have been widely found in diverse organisms including mammals, plants, and fungi as well as some prokaryotes (Vallee, 1991; Coyle et al., 2002; Cobbett and Goldsbrough, 2002). Recent studies in animals as well as plants demonstrated that MTs are not only involved in maintaining homeostasis of essential metals and metal detoxification (Cobbett and Goldsbrough, 2002; Hall, 2002; Delhaize et al., 2004), but are also implicated in a range of physiological processes, including scavenging reactive oxidant species (ROS) (Akashi et al., 2004; Wong et al., 2004), regulating cell growth, proliferation, and activity of metalloenzymes and transcription factors (Palmiter, 1998; Haq et al., 2003), involvement in metabolism of metallo-drugs, response to stress conditions, as well as potential involvement in inflammation and cell apoptosis, and even acting as possible cellular redox sensors (Vasak and Hasler, 2000; Fabisiak et al., 2002).

The first MT identified in plant was the wheat EcMT protein in 1987 (Lane et al.), and since then more than 140 MT sequences have been recorded from various species (Guo et al., 2003; Zhou et al., 2005). With the completion of the genome sequence analysis, a recent report indicated that Arabidopsis contains an MT gene family with seven distinct active MT genes, suggesting gene amplification in a higher plant during evolution (Guo et al., 2003). Plant MT proteins have been historically divided into three major classes on the basis of the arrangement of cysteine residues in their sequences (Tommaso et al., 2000; Zhou et al., 2005). Class I MT proteins are characterized by two Cys-rich domains separated by a central cysteine free spacer, and display cysteine arrangement patterns similar to their mammalian counterparts but contain unusually long spacer regions (Vallee, 1991; Coyle et al., 2002). Class II is represented by the wheat EcMT protein in which cysteine residues are grouped into three cysteine-rich domains separated by 10 to 15 residues (Lane et al., 1987; Zhou et al., 2005). Class III consists of phytochelatins, enzymatically synthesized peptides with a poly(g-Glu-Cys)-glycine structure (Cobbett and Goldsbrough, 2002). Interestingly, most of the deduced products of MT genes found in both monocots and dicots belong to class I. Therefore, class I MT proteins in plants are 
subdivided into several types based on the contribution and location of the cysteine residues.

The structures and regulation of MT genes have been well studied in many organisms including humans, mice and yeast (Haq et al., 2003). In animals, MT genes are present as gene families, the members of which are regulated differentially and have overlapping but distinct functions (Palmiter, 1998; Coyle et al., 2002; Haq et al., 2003). Metal ion regulation of MT genes is mediated by metal-responsive element (MRE) sequence motifs contained in their promoters. MRE-binding transcription factors (MTF) that bind specifically to these MREs have been characterized from humans, mice, Drosophila and yeast, and have been shown to be essential for heavy metal response and to be involved in some tissue development and physiological reactions (Palmiter, 1998; Coyle et al., 2002; Haq et al., 2003). However, the exact signaling pathways for transcriptional activation remain to be established. On the other hand, the 5'-upstream regions of MT genes have been isolated from several plant species (Chatthai et al., 2004; Fukuzawa et al., 2004; Lü et al., 2006), but MRE core sequences have not been identified in the promoters of these plant MT genes. Southern blot analysis and cDNA isolation in Arabidopsis and other plant species have indicated that MT genes are also present as gene families in plants (Giritch et al., 1998; Liu et al., 2002; Guo et al., 2003). MTs in different individual plant species appear to be grouped into a smaller, diverse gene family. There are at least two different MTs in most plant species tested, and a recent study reports that Arabidopsis harbors an MT gene family with seven distinct active MT genes (Guo et al., 2003).

The expression pattern analysis of MT genes can help to reveal their possible biological functions. Organ specificity and developmental dependence have been reported for MT genes in many plant species. For example, the pea PsMT transcript has been found to be more abundant in roots compared to leaves and seeds (Evans et al., 1990), and the wheat EcMT and the rice OsMT-II- $1 a$ genes are preferentially expressed in developing seeds (Lane et al., 1987; Zhou et al., 2005), while a soybean MT gene is more strongly expressed in leaves than in roots (Kawashima et al., 1991). In Arabidopsis and Vicia faba leaves, the RNA expression of MTs is predominant in trichomes (Foley and Singh, 1994; Garcia-Hernandez et al., 1998), while a rice MT gene was highly expressed in stem nodes (Yu et al., 1998). Moreover, the expression of MT genes has been found to be induced by ethylene in Sambucus nigra (Coupe et al., 1995) and to increase during senescence in Arabidopsis (Garcia-Hernandez et al., 1998; Navabpour et al., 2003), and in kiwifruit and pineapple the expression of MT genes is confined to specific stages of fruit development (Ledger and Gardner, 1994; Moyle et al., 2005). In all cases, the differential expression of MT genes strongly suggests that each MT isoform may have specialized functions in different tissues. Some of the functions proposed for plant MTs include a role during development (Lane et al., 1987; Ledger and Gardner, 1994;
Zhou et al., 2005), in senescence (Garcia-Hernandez et al., 1998; Yu et al., 1998; Navabpour et al., 2003), and in protection against oxidative stress (Akashi et al., 2004; Wong et al., 2004; Gisela et al., 2004).

The completion of the rice genome sequence analysis (http/ /rgp.dna.affrc.go.jp) and the annotation of its 37,500 genes have focused our attention on molecular analyses of the MT gene family in rice. To gain a comprehensive understanding of the rice MT gene family, we explored the presence of MT genes in the rice genome, and investigated the expression patterns of all rice MT genes at the tissue level using northern blot analysis. These analyses focused mainly on the gene cloning, expression patterns, genome localization, gene structure, exon/intron organization, upstream regulatory element, and evolutionary relationship of each member within the rice MT gene family. It is expected that this work will provide an important insight into the rice MT gene family. Therefore, our results reported here will provide a framework for continued studies to gain an understanding of the structure-function relationship between members of this OsMT gene family.

\section{Materials and Methods}

Database searching and sequence analysis. The sequence data used in this study were collected using the keyword "metallothionein" and a query search in the DDBJ/EMBL/GenBank database using the two known MT gene sequences from rice (U77294) and wheat (P30570). DNA sequences homologous to ricMT were then used to screen the RGP database (http://rgp.dna.affrc.go.jp) again to confirm the target gene. Gene positions on rice chromosomes were determined using NCBI (http://www.ncbi.nlm.nih.gov/BLAST) and CBI databases (http//www.btn.org.cn). The deduced amino acid sequences of ORFs were initially aligned using the program ClustalX (version 1.8) with default gap penalties (Thompson et al., 1997). A phylogenetic tree was constructed from amino acid sequences of deduced rice MT proteins by neighbor-joining algorithms and then displayed by TreeView (V 1.6.6) (Page, 1996).

Intron delimitation of target genes within the genomic DNA sequence was made by comparing the cDNA sequence and its genomic DNA sequences derived from the available RGP database. About 1500-2000 bp upstream of the translation initiation codon ATG was chosen as the upstream regulatory region for cisregulatory element analysis. Sequences of the known regulatory elements were used to search the PLACE database (http:// www.dna.affrc.go.jp/ htdocs/PLACE/) (Higo et al., 1999) for regulatory cis-element analysis of the target gene.

Isolation of total RNA and the cDNAs. Rice (Oryza sativa L. cv. Zhonghua 10) was grown in a greenhouse at $26^{\circ} \mathrm{C}$. Samples of roots, stems, leaves, sheaths and rachises, glumes before pollination, 2-day-old glumes after pollination and 15-day-old seeds were harvested from mature plants. All prepared materials were immediately frozen in liquid nitrogen and stored at $-80^{\circ} \mathrm{C}$ until use. Total RNA was extracted from various rice tissues using an RNeasy Plant Mini Kit (Qiagen), in accordance with the instructions 
provided by the manufacturer. In order to isolate the cDNAs, total RNA from different tissues were reverse transcribed with MMLV reverse transcriptase (BioLabs) and oligo(dT) following the enzyme manufacturer's instructions. The resulting cDNAs were used as a template for PCR amplification with gene-specific oligonucleotide primers. The amplified fragments were isolated, gel-purified using the GeneClean III kit (Bio 101), cloned into a pGEM-T easy plasmid vector (Promega), and then the inserts were sequenced entirely on both strands using a BigDyeTM Terminator Cycle Sequencing Ready Reaction Kit (Perkin Elmer, Germany) on an ABI PRISMTM 377 DNA Sequencer.

Northern blotting. Total RNA extracted from various tissues of rice were subjected to electrophoresis in $1.2 \%$ formaldehyde $(2.2$ M) agarose gels in $1 \times$ MOPS buffer, and blotted to a nylon membrane (Hybond $\mathrm{N}^{+}$, Amersham) using the manufacturer's recommended techniques. Hybridizations were performed by ${ }^{32} \mathrm{P}-$ labeled cDNA probes of their $3^{\prime}$ untranslated regions with random labeled probes (Prime-a-Gene random labeling system, Promega), overnight at $65^{\circ} \mathrm{C}$, in a hybridization buffer containing $5 \times \mathrm{SSC}$, $5 \times$ Denhardt's solution, $0.5 \%$ SDS and $100 \mathrm{mg} / \mathrm{ml}$ heat-denatured salmon sperm. After washing the membrane once in $2 \times \mathrm{SSC}$ and $0.1 \% \mathrm{SDS}$ at room temperature for $10 \mathrm{~min}$, and twice in $1 \times \mathrm{SSC}$ and $0.1 \%$ SDS at $65^{\circ} \mathrm{C}$ for $30 \mathrm{~min}$, the filters were exposed to X-ray films at $-80^{\circ} \mathrm{C}$.

\section{Results and Discussion}

The MT gene family in rice. We have previously reported the isolation of two rice cDNAs encoding typical MTs and their expression in different rice tissues and in response to various exogenous factors (Yu et al., 1998; Zhou et al., 2005). To gain insight into the size of the MT gene family in the rice genome, the cysteine-rich signature sequences of two reported MT proteins from rice (U77294) and wheat (P30570) were used to screen the DDBJ/EMBL/GenBank database. Searching the rice genome resulted in the identification of two additional open-reading frames encoding proteins closely related to the nine previously described MTs (Hsieh et al., 1995; Yu et al., 1998; Wong et al., 2004; Zhou et al., 2005), thus increasing the total number of MT gene family members to eleven. Accession numbers and chromosome locations are shown in Table 1. The nearly complete genomic sequence of Oryza sativa has been released, covering more than $95.3 \%$ of the 430-Mb genome (International Rice Genome, 2005). The remaining $20 \mathrm{Mb}$ of the genomic sequence is expected to cover mainly rDNA repeat units and centromeric and telomeric regions, as well as other regions of complex sequence structure that are unlikely to harbor many coding sequences. Thus, we conclude that the 11 rice MT genes identified in this study are likely to represent the actual members of this family. The results show that rice MT genes constitute a gene family in rice, which is larger than that in other plant species tested (Giritch et al., 1998; Liu et al., 2002; Guo et al., 2003), indicating that the number of MT gene family members varies in different species.

The rice MT gene family was classified into two classes and class I was further divided into four types based on sequence similarities and phylogenetic relationships. The genes were named OsMT-I-1a to OsMT-I-4c and OsMT-II- $1 a$ (Table 1), in which the initials of the genus and species are followed by the general abbreviation MT followed by the class and type number as well as the gene letter (e.g., the first MT gene of class I type 1 of rice, Oryza sativa, is called OsMT-I-1a). Only one of the deduced products of this gene family, OsMT-II-1a, is representative of class II, in which the arrangement pattern of cysteine residues is similar to the Ec protein from wheat (Fig. 1B) (Lane et al., 1987). The deduced protein contains 17 cysteine residues arranged into three groups of 6,6 and 5 cysteines, with 13-15 amino acids separating the three groups (Table 1, Fig. 1B). Almost all members of the OsMT gene family belong to class I, in which cysteine residues are

Table 1. Metallothionein genes in rice

\begin{tabular}{|c|c|c|c|c|c|c|}
\hline Name & Accession $^{\mathrm{a}}$ & $\begin{array}{l}\text { Chromosome }^{\mathrm{b}} \\
\text { (location) }\end{array}$ & $\begin{array}{l}\text { Length } \\
\text { (aa) }\end{array}$ & $\begin{array}{l}\text { Number of } \\
\text { cysteines }\end{array}$ & $\begin{array}{c}\text { Cys } \\
\text { Content }(\%)\end{array}$ & $\begin{array}{l}\text { Spacer } \\
\text { (aa) }\end{array}$ \\
\hline OsMT-I-1a $(\operatorname{rgMT}-1)^{\mathrm{c}}$ & U43529 & $11(22867248-22867890)$ & 74 & $6+6$ & 16 & 43 \\
\hline OsMT-I-1b(OsMTlc) & AK059587 & $3(10856009-10856787)$ & 72 & $6+6$ & 17 & 41 \\
\hline OsMT-I-2a(OsMT2-1) & D15602 & $1(2690331-2691774)$ & 82 & $8+6$ & 17 & 43 \\
\hline OsMT-I-2b (ricMT) & AB002820 & $1(47069952-47070629)$ & 80 & $8+9$ & 21 & 37 \\
\hline \multirow{3}{*}{$\begin{array}{l}\text { OsMT-I-2c(OsMT2b) } \\
\text { OsMT-I-3a (OsMT3) } \\
\text { OsMT-I-3b(OsMT3b) }\end{array}$} & U77294 & $5(610674-611550)$ & 84 & $8+9$ & 20 & 40 \\
\hline & AF001396 & $1(5877260-5878381)$ & 62 & $4+6$ & 16 & 36 \\
\hline & AF009959 & $5(6291741-6293946)$ & 65 & $4+6$ & 15 & 33 \\
\hline OsMT-I-4a(OsMTIb) & AK103445 & 12 (19026791-19027727) & 78 & $7+6$ & 17 & 44 \\
\hline \multirow{3}{*}{$\begin{array}{l}\text { OsMT-I-4b* } \\
\text { OsMT-I-4c* } \\
\text { OsMT-II-1a(OsMT4) }\end{array}$} & BE039194 & $12(23346012-23345084)$ & 79 & $6+6$ & 15 & 47 \\
\hline & BE039221 & $12(23476980-23477851)$ & 78 & $6+6$ & 15 & 46 \\
\hline & Ak102405 & $10(20660589-20661325)$ & 87 & $6+6+5$ & 20 & $13+15$ \\
\hline
\end{tabular}

${ }^{\mathrm{a}}$ GenBank Accession number for each gene; ${ }^{b}$ Location indicates the position of the first and last nucleotide of each gene in the chromosome; 'Brackets indicate previously designated names. Asterisks indicate new metallothioneins found from database search. 
(A)

OsMT-I-1a
OsMT-I-1b
OsMT-I-2a
OsMT-I-2b
OSMT-I-2c
OsMT-I-3a
OSMT-I-3b
OSMT-I-4a
OSMT-I-4b
OSMT-I-4c

OSMT-I-1a OsMT-I-1b OSMT-I-2a OSMT-I- $2 b$ OSMT-I-2c OSMT-I-3a OsMT-I-3b OsMT-I- 4 a OSMT-I-4b OSMT-I-4C (B) Wheat Ec AtMT $4 a$

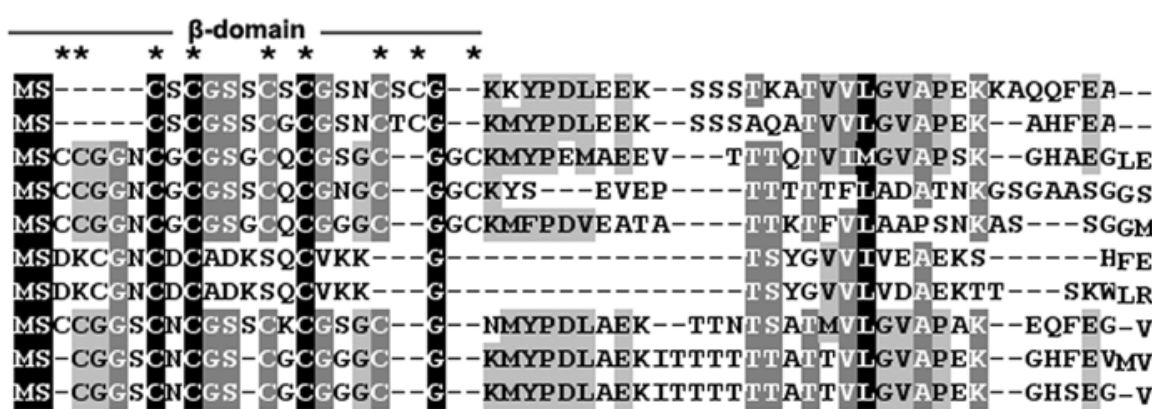

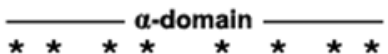

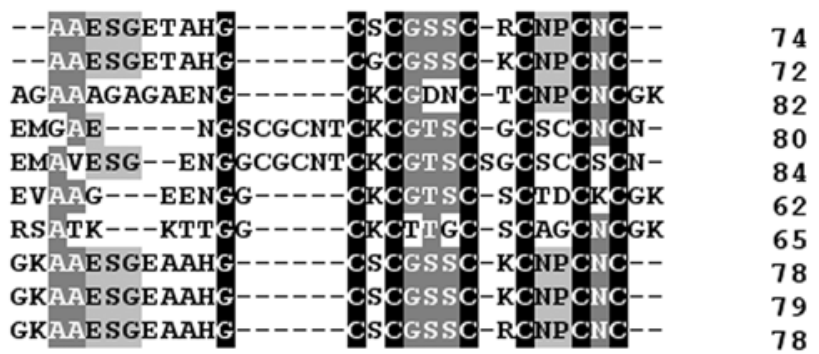

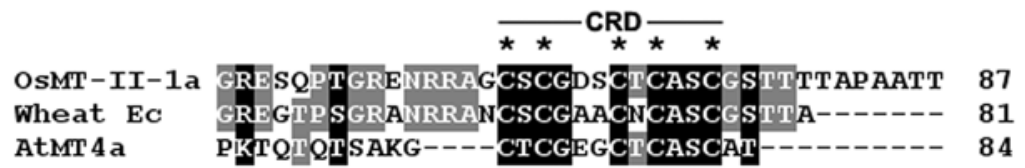

(C)

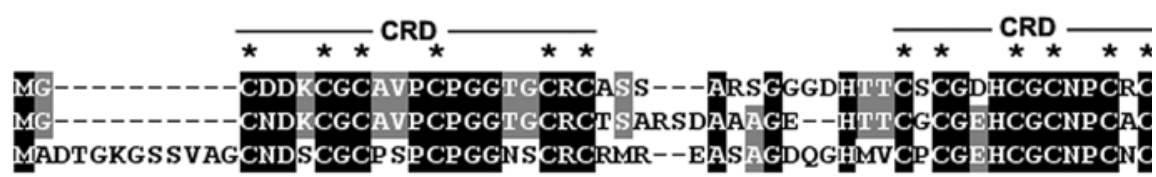

$$
\overline{\star \star} \underset{\star}{\mathrm{CRD}} \overline{\star \star}
$$

Wheat Ec

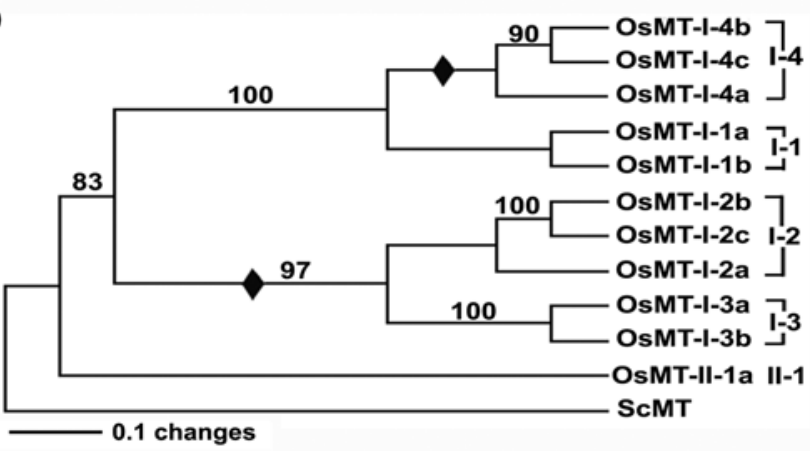

Fig. 1. Multiple alignments (A and $B$ ) and a phylogenetic tree (C) were constructed from the deduced amino acid sequences of the eleven-member OsMT gene family. Sequences were aligned with the ClustalX 1.8 program. The asterisks above each sequence alignment represent the conserved cysteine residues (C) in all putative MT proteins. Dashes indicate gaps in the sequence to allow for maximal alignment. Gray shades in varying degree are used to designate amino acids conserved at a given position in the multiple sequence alignment to at least $50 \%$. Dark shading with white letters, medium gray shading with white letters, and light gray shading with black letters reflect $90 \%, 70 \%$, and $50 \%$ sequence conservation, respectively. CRDs above the class II MT protein sequences represent cysteine-rich domains. An alignment for class I OsMT proteins is shown in (A), while the alignment of class II OsMT, OsMT-II-1a, together with the sequences of other class II MT proteins (Wheat Ec:P30570; AtMT4a: p93476) is displayed in (B). The phylogenetic tree (C) was constructed based on 1000 neighbor-joining trees from bootstrapped data sets. The MT sequence of $S$. cerevisiae (ScMT, accession No. AAA66061) was used as an outgroup, and postulated intron gains are indicated by filled diamonds. 
grouped into two cysteine-rich domains (Fig. 1A). Unlike plant class II proteins, however, one major distinction is that 33-47 amino acids link the two cysteine-rich domains (Table 1, Fig. 1A). The class I members are further divided into four types based on the cysteine arrangement patterns in their aminoand carboxy-terminal (Fig.1). Obviously, the phylogenetic analysis with strong bootstrap support for the monophyly of each clade (Fig. 1C) also supports this classification system.

The deduced amino acid sequences from 11 OsMT genes ranged from 62 amino acids (OsMT-I-4a) to 87 amino acids (OsMT-II-1a) (Table 1, Fig. 1). Unlike the conserved cysteine patterns in mammalians (Coyle et al., 2002; Haq et al., 2003), the cysteine arrangement patterns in plants seem more complicated, although 11 putative OsMT proteins all contain cysteine-rich signature motifs. The overall gene sequence similarity varies substantially between individual pairs of sequences, from $96 \%$ to less than $15 \%$ identity (Fig. 1), and the content of cysteine residues also varied from $15 \%$ to $21 \%$ (Table 1, Fig. 1), which is lower than for mammalian MTs $(\sim 30 \%)$. Despite this diversity, cysteine-rich conserved motifs are clearly present in all OsMT amino acid sequences (Fig. $1 \mathrm{~A}$ and $\mathrm{B})$.

The chromosomal locations of MT genes have been determined in some plant species (Giritch et al., 1998; Liu et al., 2002; Guo et al., 2003), as the localization of MT genes could help to provide valuable information for the genetic study of plant MT proteins. Analysis of the chromosomal distribution of OsMT genes revealed that three genes in type 4 are distributed in the same chromosome but are not closely linked, while other genes from the same type are located on different chromosomes as single copy (Table 1). This dispersed pattern of OsMT genes is principally similar to that of tomato (Giritch et al., 1998) and Arabidopsis genomes (Guo et al., 2003) where they are also are mainly distributed as single copy over the whole genome. While most MT genes of Arabidopsis are located close to the telomeres, and in tomato at least two members are also found close to the centromeres of chromosomes, none of the OsMT genes are found close to the centromeres. A similar dispersion pattern of MT genes has been found for Candida glabrata (Mehra et al., 1990) and Caenorhabditis elegans (Freedman et al., 1993). This is in clear contrast to the situation described for mammals where the functional MT genes are tightly linked to each other on chromosomes 8 and 16 in mice and humans, respectively (Palmiter et al., 1992). Whether the different organization of MT gene families in plants, yeast and lower animals is relevant to their function and/or evolution remains to be investigated.

As mentioned above, three genes of type 4 are located on the same chromosome and distributed within a 140-kb fragment of genomic DNA (Table 1). Furthermore, they share a high degree of sequence similarity, and their closeness is also reflected in the terminal subgroups of the phylogenetic tree (Fig. 1C). These results imply that the type 4 genes may have evolved from gene duplications of more recent unequal recombination events. Similar features can also be found in cotton and Arabidopsis gene families (Hudspeth et al., 1996; Guo et al., 2003). In contrast, the functional MT genes in mammals are tightly linked (Palmiter et al., 1992). Therefore, linked organization of MT genes seems to be a characteristic associated solely with mammals.

Differential expression of OsMT genes in rice tissues. Although several rice MT genes were reported to be expressed at very high levels in different rice tissues (Hsieh et al., 1995; Yu et al., 1998; Wong et al., 2004; Zhou et al., 2005), no studies have yet provided a comprehensive description of the expression of the OsMT gene family in rice. In the present study, we relied primarily on RNA blot hybridization to examine the tissue-specific expression of OsMT genes in different tissues of rice, including roots, stems, rachises, glumes before pollination, 2-day-old glumes after pollination, 15-day-old seeds, young leaves, mature leaves, old leaves, young sheaths, mature sheaths and old sheaths (Fig. 2). It is worth pointing out that there is a high encoding sequence similarity within some OsMT genes, and their hybridizations were therefore performed with ${ }^{32} \mathrm{P}$-labeled cDNA probes of their 3' untranslated regions.

Northern analysis showed that accumulation of OsMT gene transcripts not only was associated with different tissues and stages of development, but the expression pattern of each OsMT gene member also differed. As shown in Fig. 2, OsMT$I-3 a, O s M T-I-4 a$ and OsMT-I-4b mRNA were expressed at a high level only in roots, but at a very low level in leaves and/ or sheaths. Furthermore, a similar pattern was also observed in the case of $O s M T-I-1 a$ and $O s M T-I-1 b$, which were principally expressed in old leaves (and old sheaths for the former), while the expression levels were very low or barely detectable in other tissues. In addition, the level of OsMT-I-3b mRNA was noticeably higher in rachises, leaves and sheaths, but was barely detected in the roots, stems and glumes. However, there was a constitutive accumulation of type 2 gene transcripts (except in roots) for $O s M T-I-2 a$, but the accumulation patterns among the other three members were different. The highest expression of $O s M T-I-2 c$ was detected in the stems and reproductive tissues such as rachises, glumes and seeds. It should be also noted that only type 2 genes within the OsMT gene family could be expressed at high levels in the stems. The expression of OsMT-I-4c exhibited the highest levels in rachises, leaves and old sheaths, and relatively low levels in inflorescences, development seeds and young or mature sheaths, whereas no transcripts of the OsMT-I-4c were detected in roots and stems.

With respect to class II genes, previous studies indicated that expression of the wheat Ec MT gene is restricted to developing seeds, and it might provide a mechanism for storing zinc, which is required during germination (Lane et al., 1987). Our results show that OsMT-II-1a mRNA is also present mainly in 2-day-old glumes after pollination and 15day-old seeds (Fig. 2). This suggests the OsMT-II-1a protein 


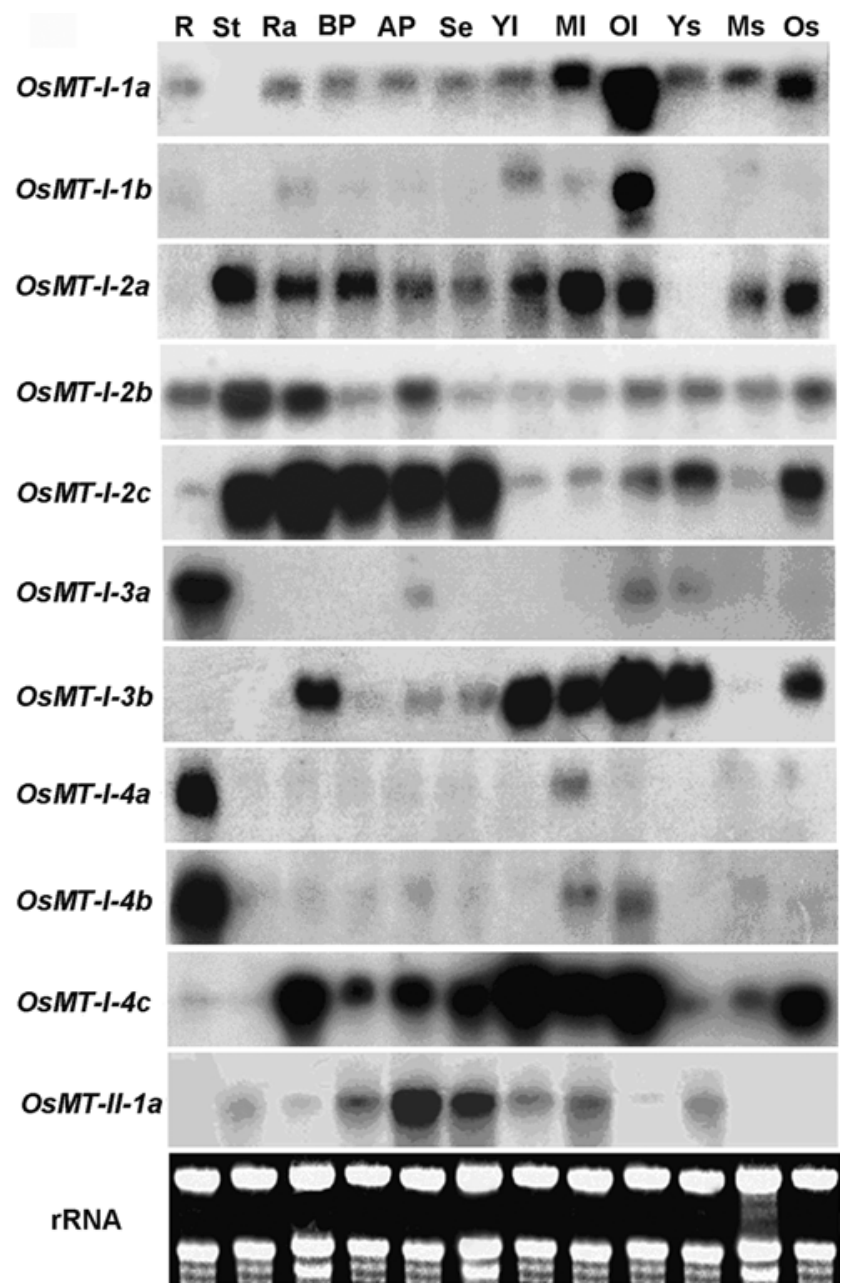

Fig. 2. Expression of OsMT genes in mature rice plants. Total RNAs were prepared from various tissues, including roots (R), stems (St), raches (Ra), inflorescences before pollination (BP), 2day-old inflorescences after pollination (AP), 15-day-old seeds (Se), young leaves ( $\mathrm{Yl}$ ), mature leaves (Ml), old leaves $(\mathrm{Ol})$, young sheaths (Ys), mature sheaths (Ms) and old sheaths (Os). RNA $(10 \mu \mathrm{g})$ was loaded in each lane except $5.0 \mu \mathrm{g}$ RNA for the lane of the stems with OsMT-I-2b. The ethidium bromidestained rRNA bands are shown as a loading control. Exposure times: 72 hours for OsMT3a and OsMT5b; 24 hours for others.

not only plays a role in developing seeds similar to that of Ec protein, but it might also be involved in providing metal ions to germinating pollen grains, consistent with it being barely transcribed in unpollinated glumes. Interestingly, we found that OsMT-I-2b mRNA (but not OsMT-I-2c and OsMT-I-4c mRNA) was also more abundant in 2-day-old glumes after pollination, and was expressed only slightly in sheaths. Clearly, expressions of several OsMT genes from class I have also been observed in reproductive development, suggesting these OsMT proteins might also be involved in accumulation and storage of metals in reproductive development. If so, they may play an important role in determining the concentrations of metals in grains. As more attention is paid to the nutritional composition of foods as opposed to simple caloric value, these MT proteins may provide one mechanism to manipulate metal concentrations in seeds (Lucca et al., 2001) and thus produce nutritionally enhanced grains.

One interesting phenomenon is the dramatic increase in some mRNA levels in senescing leaves and/or sheaths, such as OsMT-I-1a, OsMT-I-1b, OsMT-I-2a and OsMT-I-4c, which has been displayed in many plant MT genes (Cobbett and Goldsbrough, 2002; Navabpour et al., 2003; Gisela et al., 2004). This suggests that these OsMT proteins might serve as a chaperone for long-distance transport of essential transition metals from senescing tissues to young or eugonic tissues in order to maintain the normal growth and development of plants, or serve as a phytochelatin of toxic metals to maintain the homeostasis of metal ions and prevent the damage that heavy metals can cause through the production of reactive oxygen species to membranes, proteins, and nucleic acids (Cobbett and Goldsbrough, 2002; Navabpour et al., 2003; Gisela et al., 2004). However, OsMT-I-3a, OsMT-I-4a and OsMT-I-4b mRNA were expressed at a high level only in roots, which suggest there is another way to avoid the damage by vacuolar sequestration of metal ions (Cobbett and Goldsbrough, 2002). Furthermore, type 2 genes and OsMT-I$4 c$ are expressed in an almost constitutive pattern (Fig. 2), which provides a hint that these genes may play a background role in certain homeostatic mechanisms, whereas highly induced OsMT genes in some special tissues and with the treatments of different metals and exogenous factors are probably adaptive to various development processes or environmental stresses (unpublished data). Clearly, the nature of MT proteins under varying conditions will be a fruitful and important area of future investigation.

As described above, the results provided a comprehensive description of the expression profiles of all members from the OsMT gene family in mature rice plants. Although each member of this family differs not only in sequence but also in expression, the diverse patterns of expression of different OsMT members suggest that these isoforms may have different functions they perform in specific tissues or different developmental stages.

Exon/intron organization of the OsMT gene family. As shown in Fig. 3, OsMT genes possess relatively simple structures and show a very striking bimodal distribution of introns, with type 2-4 containing two introns and type 1 and class II only having a single intron. Five MT genes identified from the Arabidopsis genome also contain two exons interrupted by a single intron (Zhou and Goldsbrough, 1995). Comparing intron positions within all OsMT gene sequences, two independent intron insertion events appear to have occurred during the course of OsMT gene evolution. The most widespread, and therefore probably the oldest, is the first intron, which was found to have a conserved position in all of the 11 OsMT gene sequences (Fig. 3A and 3B). On the other hand, the second intron is found only within types $2-4$; a single clear case of the 
(A)

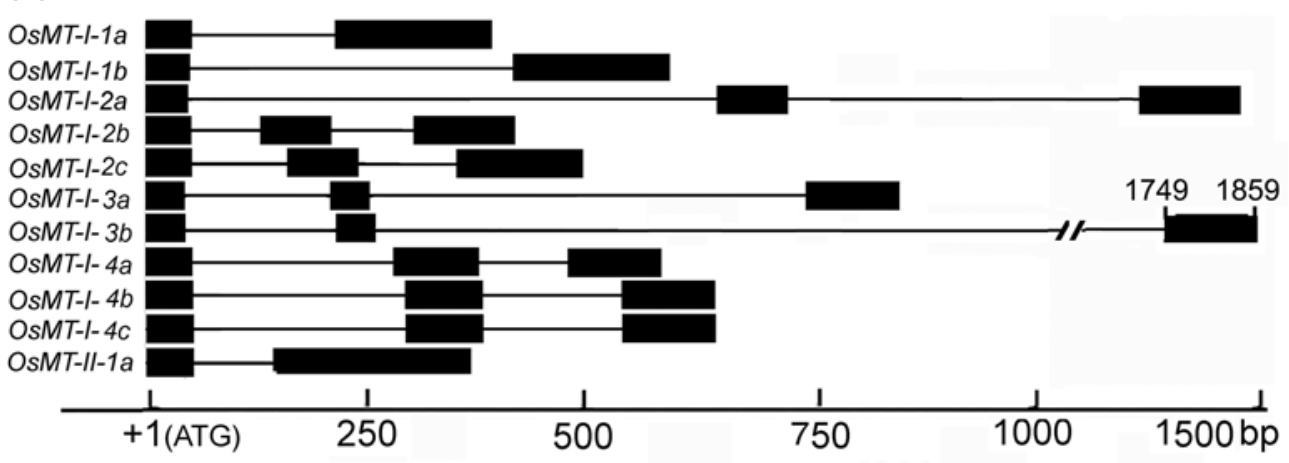

(B)
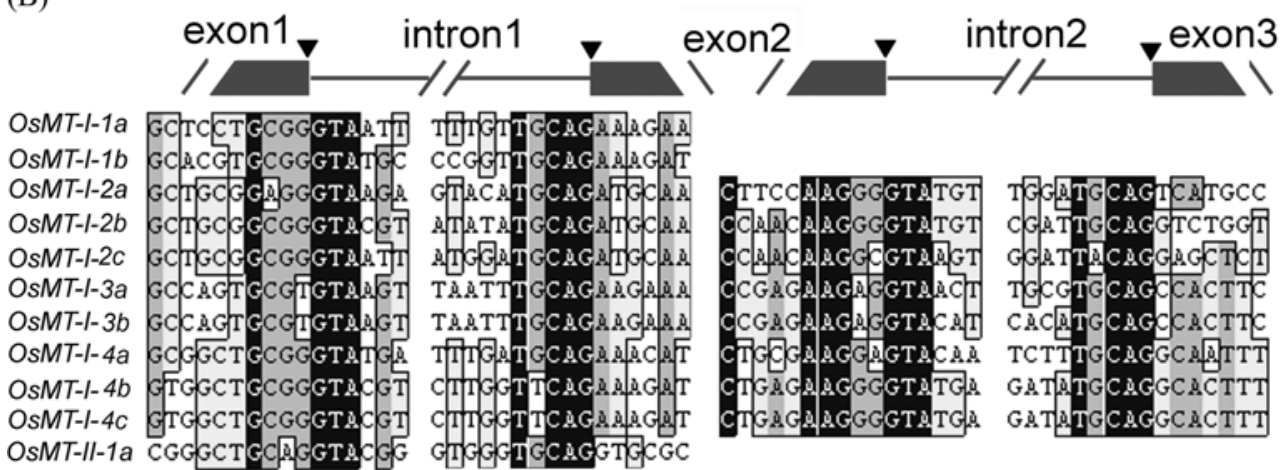

Fig. 3. A schematic diagram of the exon/intron structures of the OsMT genes. (A) The conserved exon-intron structures of all member genes in the OsMT family. Boxes and lines indicate exons and introns, respectively. (B) Nucleotide sequences around the splicing sites of the eleven members of the OsMT gene family. Gray boxes indicate the exon regions, gray lines represent the intron regions, and black inverted triangles indicate the splicing positions.

second intron gain is seen in types 2-3 (Fig. 3A). Similarly, the presence of the second intron in type 4 may also have arisen from an intron gain that occurred in the duplication event separating type 4 from type 1 (Figs. 1 and 3). These results suggest that OsMT genes with two introns are derived from those of one intron by insertions, and that the presentday OsMT genes possibly evolved through endoduplication followed by the addition of an intron.

The first intron in type 1, 3 and 4 OsMT genes disrupts the codon after the last cysteine codon in the N-terminal cysteinerich domain. Unlike the position of the first intron in types 1, 3 and 4 , the first intron in type 2 and class II OsMT genes lies in the same relative position before the end of the N-terminal cysteine-rich domain (Fig. 3B). However, the second intron in genes encoding type 2, 3 and 4 OsMT proteins lies in the middle of the spacer (Fig. 3). Interestingly, in dicot Arabidopsis, the second intron in type 2 genes is lost (data not shown). These facts indicate that the first intron is located close to the end of the N-terminal cysteine-rich domain and the position of this intron varies in genes encoding different OsMT classes and types (further discussion below). Furthermore, apart from the difference between type 2 MT genes from monocots and dicots, the intron position in plant MT genes also provides some additional support for the classification system described above.

Further investigation of the sequences around the splicing sites of these genes provided us with some evidence of the inherent relationship between them. Although the identity of the nucleic acid sequences of these eleven OsMT genes is only about $33 \%$, the regions around the splicing sites exhibit high homology (Fig. 3B). The conserved sequences around the splicing site and the fixed splicing position strongly imply a consanguineous relationship among the OsMT gene family. Otherwise, all sequences bordering the introns/exons conform to the GT/AG but not to the AT/AC rule (Mount, 1996) for splice junctions (Fig. 3B). Therefore, analysis of the splicing process will provide us with more information about the evolutionary relationship among those eleven proteins.

In all eleven OsMT proteins, the first splicing position appears near the end of the â domain (Fig. 3). The splicing mechanism is highly conserved in this OsMT gene family. It seems that the first splicing step determines the â domain of OsMT proteins. Although the MT genes in A. thaliana commonly contain two exons and one intron, which make it significantly different from those in rice, the position of the first splicing site is highly conserved: it usually occurs close to the end of the $\beta$ domain of the gene products. Recent studies showed that the $\beta$ domains of plant MTs have been predicted to be able to 
Table 2 Putative cis-element sequences in 5'-flanking regions in the OsMT gene family

\begin{tabular}{|c|c|c|c|c|}
\hline Symbol & Name & Sequence & Description & Ref. \\
\hline Red rectangle & MRE & TGCRCNC & metal responsive element & [Lü ] \\
\hline Green rectangle & CRE & CTGCCA & copper responsive element & [Lü ] \\
\hline $\mathrm{A}$ & TATA box & TATAA & eukaryotic promoter for RNA polymerase II & [Lü ] \\
\hline $\mathrm{B}$ & CAAT box & CCAAT & transcription factor $\mathrm{CTF} / \mathrm{NF} 1$ binding site & [Lü ] \\
\hline $\mathrm{C}$ & GC box & GGGCGG & transcription factor SP1 binding site & [Lü ] \\
\hline \multirow{4}{*}{$\mathrm{D}$} & \multirow{4}{*}{ AuxRE } & TGTCTC & "auxin response factor" binding site & [Ulmasov] \\
\hline & & CATATG & involved in auxin responsiveness & {$[\mathrm{Xu}$ N.] } \\
\hline & & ACTTTA & required for auxin induction & [Baumann] \\
\hline & & KGTCCCAT & auxin responsive element of pea PS-IAA $4 / 5$ gene & [Ballas] \\
\hline \multirow{2}{*}{$\mathrm{E}$} & \multirow{2}{*}{ ABARE } & ACACNNG & ABA responsive element & [Kim] \\
\hline & & CATGCA & $\mathrm{ABA}$ responsive element & [Ezcurra] \\
\hline \multirow{2}{*}{$\mathrm{F}$} & \multirow{2}{*}{ ERE } & AWTTCAAA & ethylene responsive element in tomato & [Montgomery] \\
\hline & & GCCGCC & core of GCC-box as ethylene-responsive element & [Zhou G.K.] \\
\hline $\mathrm{G}$ & GRE & TAACGTA or TAACAGA & "gibberellin-responsive element" in rice & [Sutoh] \\
\hline $\mathrm{H}$ & ARE & TGACNNNGC & antioxidant-responsive element & [Zhou G.K.] \\
\hline I & HSRE & CTNGAANNTTCNAG & heat shock-responsive element & [Gurley] \\
\hline $\mathrm{J}$ & LTRE & CCGAC & low temperature responsive element & [Zhou G.K.] \\
\hline $\mathrm{K}$ & SSRE & GCCGCGC or GCCGGCC & sucrose starvation responsive element & [Bai] \\
\hline $\mathrm{L}$ & OSE & TACTAT & organ-specific element & [Lü] \\
\hline $\mathrm{T}$ & TIS & YYYAYYA & transcription initiation site & [van der Meer] \\
\hline
\end{tabular}

Note: Symbols used in the sequences are listed as follows: N: A, C, G or T; R: A or G; W: A or T; Y: C or T; K: G or T.

function independently (Lu et al., 2003; Bilecen et al., 2005). Therefore, the conserved splicing position after a long period of evolution of millions of years might imply an important functional role of the $\beta$ domain. It is no doubt an effective mechanism to reduce the risk of producing malfunctioned protein products by splicing close to the functional domain. Even if there is something wrong with the splicing process in some situations, the first spliced exon may have a chance to be translated, in some unusual ways, into a functional peptide and thus compensate for the harm caused by the mis-splicing. Indeed, in some low-grade species such as fungi (He et al., 2002) and crab (Kagi, 1993), the functional MT proteins only contain $\beta$ domains or â-like domains. It is supposed that the higher plants have developed a class of more complex MT proteins and will start a backup program to produce relatively simple but functional products, as their ancestors did, when placed in an abnormal splicing situation. The case in other plants remains unclear, although we can reasonably hypothesize that this splicing rule is common in almost all plant MT genes based on observations from the two model plant species.

Interestingly, the intron position can also affect the specific improvement of intron splicing and increase the levels of mRNA (Snowden et al., 1996). The effects of the intron presence are also supported by several lines of studies (Donath et al., 1995; Xu et al., 1995; Li et al., 1995; Fu et al., 1995). We also noted that in sea urchin embryos, MT gene expression in specific cell types is regulated by the elements in its first intron (Bai et al., 1993), and in rice, the expression of GUS was enhanced by the presence of the $\operatorname{rgMT}$ (OsMT-I- 1a) intron (Hsieh and Huang, 1998). Therefore, whether the leading intron is involved in the control of spatio-temporal expression remains a valuable question for investigation. It is also still unknown whether the second intron, separately from the first one, also regulates the expression of OsMT genes.

Upstream regions of the OsMT genes. The most conspicuous feature of MTs is their inducibility by many chemical and physical agents acting directly or indirectly on multiple cisacting elements in the regulatory regions of MT genes (Haq et al., 2003). Generally speaking, gene transcription is controlled by both positive (activator) and negative (repressor) interactions between DNA and regulatory proteins, but the vast majority of events governing transcriptions of MT genes are of the activator type (Haq et al., 2003). In plants, however, a comparative analysis of the regulatory regions of the whole MT gene family has not been reported in the literature. Exploration of the regulatory regions of OsMT genes remains an intriguing area of high potential to enhance our understanding of MT protein function(s).

Identification of cis-regulatory elements is one of the major challenges in bioinformatics and integrates comparative, structural, and functional genomics. Promoters, an essential part for transcription of genes, occupy an important regulatory region in the control of gene expression. In consideration of the variety in length of the regulatory region in the different genes, we chose the appropriate genomic DNA sequences before the first exon of each gene as the positions of the upstream regulatory regions for cis-regulatory element analysis. 


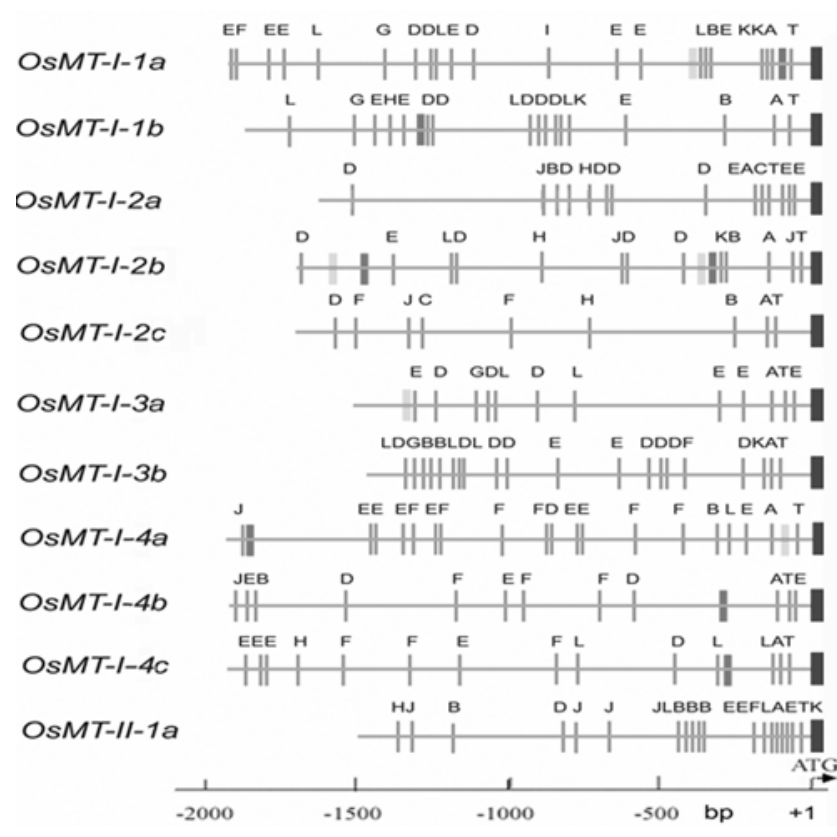

Fig. 4. Putative regulatory cis-elements in the OsMT gene family. The relative positions of cis-elements are labeled with capital letters in the figure, and are annotated in Table 2.

The putative structure and significance of the upstream region in the OsMT gene family were described in order to reveal the putative regulatory differences within members of the OsMT gene family.

A total of fifteen kinds of cis-regulatory elements were listed (Table 2) and identified in the upstream regions of these eleven OsMT genes (Fig. 4). All of the eleven upstream sequences have a putative TATA box and transcription initiation site (TIS), which are commonly located at 104 to $190 \mathrm{bp}$ and 23 to 104 bp upstream regions from the translation site (ATG) respectively. In addition, two other popular eukaryotic regulatory elements - the CAAT box and GC box - are also present in the upstream sequences, but not all members have these boxes in their upstream sequences. For example, there is GC box in the upstream region of $O s M T-I-2 a$ and $O s M T-I-2 c$ and there is no CAAT box in the upstream region of OsMT-I-3a and OsMT-I4c (Fig. 4).

MREs have been found to widely exist in the metal regulatory region of many mammalian MT genes (Coyle et al., 2002; Haq et al., 2003). The core sequence TGCRCNC has been demonstrated to be crucial in binding the MT transcription factors and thus responsible for the activation of downstream MT gene expression under metal induction. However, their counterparts in plants are poorly understood. In very few plant species, such as rice (Lü et al., 2006), pea (Evans et al., 1990) and tomato (Whitelaw et al., 1997), some putative MRE sequences have been found in the upstream regions of MT genes. In this study, we provide the first intact information on MREs on a complete gene family scale in rice and even in plants. Our results indicate that in addition to these universal regulatory elements, we also found one or two mammalian metal responsive elements (MREs) in the upstream regions of OsMT-I-1a, OsMT-I-1b, OsMT-I-2b, OsMT-I-4a, OsMT-I-4b and $O s M T-I-4 c$. Surprisingly, not all of the upstream regions of the OsMT genes were found to possess MREs (Fig. 4). One possibility is that other transcription factors might participate in the control of MT gene transcription by binding some core sequence, or there may be some other metal-responsive elements which differ greatly from the core sequences of known MREs. In other words, there may be some other transcription factors and/or cis-regulatory elements, besides known MREs, that can be responsive to metal signals. The possibility of other metal-responsive elements has been demonstrated when we identified another metal-responsive element - copper responsive element (CRE) - in the upstream regions of the MRE-lacking genes such as OsMT-I-3a. This suggestion is consistent with results obtained by Mett et al. (1993). The $A C E 1$ gene fused to the $35 \mathrm{~S}$ promoter of the cauliflower mosaic virus and the $\beta$-glucuronidase (GUS) gene under control of a promoter containing the copper-responsive element of the CUP1 gene were both introduced into tobacco. GUS activity was induced in leaves of transgenic plants upon treatment of plants with copper. However, this induction required the presence of the $A C E 1$ gene, indicating that the yeast copper-responsive element was not recognized by endogenous plant transcription factors. Therefore, CREs and/ or other unknown MREs may serve as metal-responsive elements in these known MRE-lacking MT genes. We should point out that CREs also exist in MRE-containing genes, for example, in OsMT-I- $1 a$ and OsMT-I-2b (Fig. 4), which might strengthen the metal inducing ability in cooperation with MREs. Though some plant MT proteins have been demonstrated to play an important role in binding metal ions in vitro (Lane et al., 1987), further work is needed to provide direct experimental evidence that clarifies the relationship between the metal-binding property and the putative plant MREs.

In the regulatory regions, we also found many organ-specific elements (OSEs), antioxidant-responsive elements (AREs), gibberellin-responsive elements (GREs), auxin- responsive elements (AuxREs), ABA-responsive elements (ABAREs), ethylene-responsive elements (EREs), sugar starvation-related elements (SSREs), heat shock-responsive elements (HSREs) and low temperature-responsive elements (LTREs). Of those responsive elements, the promoter sequences in some OsMT genes possess putative ABAREs, EREs, SSREs, HSREs and LTREs, and their expression is associated with ABA, ethylene, sugar starvation, heat shock and low temperature respectively (Coupe et al., 1995; Hsieh et al., 1995; Liu et al., 2002; Fukuzawa et al., 2004; Zhou et al., 2005; Lü et al., 2006). However, with respect to OSEs, AREs, GREs and AuxREs, little is known to date about regulating OsMT gene expression. Consequently, further careful work is necessary to resolve these issues.

A series of light-induced or light-related cis-elements are abundantly present in the upstream region of some OsMT 
genes (data not shown). Since the role of MT genes in plants is unclear to date, this finding may raise the possibility that plant MT genes are involved in other distinct but unproved biological processes, such as the light induced biochemical pathway, in addition to their role in binding metal ions. Based on the analysis of cis-elements in the regulatory regions of each OsMT gene, a distinctive but overlapping system of regulation patterns may have been established for the whole OsMT gene family, and hence they probably have distinct but overlapping functions in response to developmental and environmental signals, which are similar to those in the mammalian MT gene family (Vasak and Hasler, 2000; Coyle et al., 2002). Analyses of the upstream regions in the OsMT gene family help elucidate the regulatory mechanism and facilitate identification of potential new cis-regulatory elements and transcription factors that control the expression of OsMT genes in response to the homeostasis of metals under normal growth conditions, developmental processes and other environmental stresses. Our findings demonstrate that the upstream regions of the OsMT genes possess a wide variety of cis-regulatory elements present in a model plant, and provide an important insight into the transcriptional regulation machines that operate the temporal- and spatial-specific expression and stress responses of the rice MT genes. Therefore, our results here will provide a framework for continued studies on identification of regulatory elements in OsMT promoters.

In conclusion, this study provides a comprehensive description of the gene expression, gene organization, genomic localization, and phylogenetic relationship of each member of the plant MT gene family in a single species. The results presented here can serve as a useful foundation for guiding future experimental work and understanding the structure-function relationship of the members of the OsMT gene family, and a predictive framework for exploring potential cis-elements or transcription factors for regulating expression of the OsMT gene family.

Acknowledgments We thank members of the Laboratory of Molecular Biology at Tsinghua University for many insightful discussions, and Dr. Peixiang Ni for his valuable assistance in the computer analysis. This work was supported by grants from the National Natural Science Foundation of China (30270753, 30370804), and the State Key Basic Research and Development Plan of China (2004CB117303).

\section{References}

Akashi, K., Nishimura, N., Ishida, Y. and Yokota, A. (2004) Potent hydroxyl radical-scavenging activity of drought-induced type-2 metallothionein in wild watermelon. Biochem. Biophys. Res. Com. 323, 72-78.

Bai, G., Stuebing, E. W., Parker, H. R., Harlow, P. and Nemer, M. (1993) Combinatorial regulation by promoter and intron 1 regions of the metallothionein SpMTA gene in sea urchin embryo. Mol. Cell Biol. 13, 993-1001.
Ballas, N., Wong, L. M. and Theologis, A. (1993) Identification of the auxin-responsive element, AuxRE, in the primary indoleacetic acid-inducible gene, PS-IAA4/5, of pea. J. Mol. Biol. 233, 580-596.

Baumann, K., De Paolis, A., Costantino, P. and Gualberti, G. (1999) The DNA binding site of the Dof protein NtBBF1 is essential for tissue-specific and auxin-regulated expression of the rolB oncogene in plants. Plant Cell 11, 323-333.

Bilecen, K., Ozturk, U. H., Duru, A. D., Sutlu, T., Petoukhov, M. V., Svergun, D. I., Koch, M. H., Sezerman, U. O., Cakmak, I. and Sayers, Z. (2005) Triticum durum metallothionein: isolation of the gene and structural characterization of the protein using solution scattering and molecular modeling. J. Biol. Chem. 280, 13701-13711.

Chatthai, M., Osusky, M., Osuska, L., Yevtushenko, D. and Misra, S. (2004) Functional analysis of a Douglas-fir metallothioneinlike gene promoter: transient assays in zygotic and somatic embryos and stable transformation in transgenic tobacco. Planta 220, 118-128.

Cobbett, C. and Goldsbrough, P. (2002) Phytochelatins and metallothioneins: roles in heavy metal detoxification and homeostasis. Annu. Rev. Plant Biol. 53, 159-182.

Coupe, S. A., Taylor, J. E. and Roberts, J. A. (1995) Characterization of an mRNA encoding a metallothionein-like protein that accumulates during ethylene-promoted abscission of Sambucus nigra L. leaflets. Planta 97, 442-447.

Coyle, P., Philcox, J. C., Carey, L. C. and Rofe, A. M. (2002) Metallothionein: the multipurpose protein. Cell Mol. Life Sci. 59, 627-647.

Delhaize, E., Ryan, P. R., Hebb, D. M., Yamamoto, Y., Sasaki, T. and Matsumoto, H. (2004) Engineering high-level aluminum tolerance in barley with the ALMT1 gene. Proc. Natl. Acad. Sci. 101, 15249-15254.

Donath, M., Mendel, R., Cerff, R. and Martin, W. (1995) Introndependent transient expression of the maize GapA1 gene. Plant Mol. Biol. 28, 667-676.

Evans, I. M., Gatehouse, L. N., Gatehouse, J. A., Robinson, N. J. and Croy, R. R. (1990) A gene from pea (Pisum sativum L.) with homology to metallothionein genes. FEBS Lett. 262, 2932.

Ezcurra, I., Ellerstrom, M., Wycliffe, P., Stalberg, K. and Rask, L. (1999) Interaction between composite elements in the napA promoter: both the B-box ABA-responsive complex and the RY/G complex are necessary for seed-specific expression. Plant Mol. Biol. 40, 699-709.

Fabisiak, J. P., Borisenko, G. G., Liu, S. X., Tyurin, V. A., Pitt, B. R. and Kagan, V. E. (2002) Redox sensor function of metallothioneins. Methods Enzymol. 353, 268-281.

Foley, R. C. and Singh, K. B. (1994) Isolation of a Vicia faba metallothionein-like gene: expression in foliar trichomes. Plant Mol. Biol. 26, 435-444.

Freedman, J. H., Slice, L. W., Dixon, D., Fire, A. and Rubin, C. S. (1993) The novel metallothionein genes of Caenorhabditis elegans. J. Biol. Chem. 268, 2554-2564.

Fu, H., Kim, S. Y. and Park, W. D. (1995) High-level tuber expression and sucrose inducibility of a potato Sus 4 sucrose synthase gene require 5'-flanking and 3'-flanking sequences and the leader intron. Plant Cell 7, 1387-1394.

Fukuzawa, H., Yu, L. H., Umeda-Hara, C., Tagawa, M. and Uchimiya, H. (2004) The rice metallothionein gene promoter 
does not direct foreign gene expression in seed endosperm. Plant Cell Rep. 23, 231-235.

Garcia-Hernandez, M., Murphy, A. and Taiz, L. (1998) Metallothioneins 1 and 2 have distinct but overlapping expression patterns in Arabidopsis. Plant Physiol. 118, 387397.

Giritch, A., Ganal, M., Stephan, U. W. and Baumlein, H. (1998) Structure, expression and chromosomal localisation of the metallothionein-like gene family of tomato. Plant Mol. Biol. 37, 701-714.

Gisela, M., Jordi, D., Gemma, H., Guo, W. J., Goldsbrough, P., Atrian, S. and Molinas, M. (2004) A plant type 2 metallothionein (MT) from cork tissue responds to oxidative stress. J. Exp. Bot. 55, 2483-2493.

Guo, W. J., Bundithya, W. and Goldsbrough, P. B. (2003) Characterization of the Arabidopsis metallothionein gene family: tissue-specific expression and induction during senescence and in response to cupper. New Phytol. 59, 369-381.

Gurley, W. B., Czarnecka, E., Key, J. L. and Nagao, R. T. (1986) Upstream sequences required for efficient expression of a soybean heat shock gene. Mol. Cell Biol. 6, 559-565.

Hall, J. L. (2002) Cellular mechanisms for heavy metal detoxification and tolerance. J. Exp. Bot. 53, 1-11.

Haq, F., Mahoney, M. and Koropatnick, J. (2003) Signaling events for metallothionein induction. Mutation Res. 533, 211-226.

He, H., Lu, T., Zhang, R., Zhao, N. and Liu, J. Y. (2002) A prediction for the tertiary structure of metallothionein CAP3 from Colletotrichun gloeosporioides. Prog. Biochem. Biophys. 29, 293-296.

Higo, K., Ugawa, Y., Iwamoto, M. and Korenaga, T. (1999) Plant cis-acting regulatory DNA element (PLACE) database: 1999. Nucleic Acid Res. 27, 297-300.

Hsieh, H. M. and Huang, P. C. (1998) Promoter structure and activity of type 1 rice metallothionein-like gene. DNA Seq. 9, 9-17.

Hsieh, H. M., Liu, W. K. and Huang, P. C. (1995) A novel stressinducible metallothionein-like gene from rice. Plant Mol. Biol. 8, 381-389.

Hudspeth, R. L., Hobbs, S. L., Anderson, D. M., Rajasekaran, K. and Grula, J. W. (1996) Characterization and expression of metallothionein-like genes in cotton. Plant Mol. Biol. 31, 701705 .

International Rice Genome Sequencing Project. (2005) The mapbased sequence of the rice genome. Nature 436, 793-800.

Kagi, J. H .R. (1993) Evolution, structure, and chemical activity of class I metallothionein: an overview; in Metallothionein III, Klaassen C. (ed.), pp. 29-56, Birkhauser Press Boston, USA.

Kawashima, I., Inokuchi, Y., Chino, M., Kimura, M. and Shimizu, N. (1991) Isolation of a gene for a metallothionien-like protein from soybean. Plant Cell Physiol. 32, 913-916.

Kim, S. Y., Chung, H. J. and Thomas, T. L. (1997) Isolation of a novel class of bZIP transcription factors that interact with ABA-responsive and embryo-specification elements in the Dc3 promoter using a modified yeast one-hybrid system. Plant $J$. 11, 1237-1251.

Lane, B., Kajioka, R. and Kennedy, T. (1987) The wheat-germ Ec protein is a zinc-containing metallothionein. Biochem. Cell Biol. 65, 1001-1005.

Ledger, S. E. and Gardner, R. C. (1994) Cloning and characterization of five cDNAs for genes differentially expressed during fruit development of kiwifruit (Actinidia deliciosa var. deliciosa). Plant Mol. Biol. 25, 877-886.

Li, Y. Z., Ma, H. M., Zhang, J. Y. and Hong, M. M. (1995) Effects of the first intron of rice Waxy gene on the expression of foreign genes in rice and tobacco protoplasts. Plant Sci. 108, 181-190.

Liu, P., Goh, C. J., Loh, C. S. and Pua, E. C. (2002) Differential expression and characterization of three metallothionein-like genes in Cavendish banana (Musa acuminata). Physiol. Plant 114, 241-250.

Lu, T., Liu, J. Y., Zhang, R. Q. and Zhao, N. M. (2003) Modeling rice $\operatorname{rgMT}$ as a plant metallothionein-like protein by the distance geometry and homology methods, Acta Bot. Sin. 45, $1297-1306$

Lucca, P., Hurrell, R. and Potrykus, I. (2001) Genetic engineering approaches to improve the bioavailability and the level of iron in rice grains. Theor. Appl. Genet. 102, 392-397.

Lü, S., Gu, H., Yuan, X., Wang, X., Wu, A., Qu, L. J. and Liu, J. Y. (2006) The GUS reporter-aided analysis of the promoter activities of a rice metallothionein gene reveals different regulatory regions responsible for tissue-specific and inducible expression in transgenic Arabidopsis. Transgenic Research in press.

Mehra, P. K., Garey, J. R. and Winge, D. R. (1990) Selective and tandem amplification of a member of the metallothionein gene family in Candida glabrata. J. Biol. Chem. 265, 6369-6375.

Mett, V. L., Lochhead, L. P. and Reynolds, H. S. (1993) Coppercontrollable gene expression system for whole plants. Proc. Natl. Acad. Sci. 90, 4567-4571.

Montgomery, J., Goldman, S., Deikman, J., Margossian, L. and Fischer, R. L. (1993) Identification of an ethylene-responsive region in the promoter of a fruit ripening gene. Proc. Natl. Acad. Sci. 90, 5939-5943.

Mount, S. M. (1996) AT-AC introns: an AT/ACk on dogma. Science 271, 1690-1692.

Moyle, R., Fairbairn, D. J., Ripi, J., Crowe, M. and Botella, J. R. (2005) Developing pineapple fruit has a small transcriptome dominated by metallothionein. J. Exp. Bot. 56, 101-112.

Navabpour, S., Morris, K., Allen, R., Harrison, E., A-HMackerness, S. and Buchanan-Wollaston, V. (2003) Expression of senescence-enhanced genes in response to oxidative stress. J. Exp. Bot. 54, 2285-2292.

Page, R. D. M. (1996) TREEVIEW: an application to display phylogenetic trees on personal computers. Computer Appl. Biosci. 12, 357-358.

Palmiter, R. D. (1998) The elusive function of metallothioneins. Proc. Natl. Acad. Sci. 95, 8428-8430.

Palmiter, R. D., Findley, S. D., Whitmore, T. E. and Durnam, D. M. (1992) MT-III, a brain-specific member of the metallothionein gene family. Proc. Natl. Acad. Sci. 89, 63336337.

Snowden, K. C., Buchholz, W. G. and Hall, T. C. (1996) Intron position affects expression from the tpi promoter in rice. Plant Mol. Biol. 31, 689-692.

Sutoh, K. and Yamauchi, D. (2003) Two cis-acting elements necessary and sufficient for gibberellin-upregulated proteinase expression in rice seeds. Plant J. 34, 636-645.

Tommaso, G., Lucia, N., Bianca, E. M., Sonia, T. and Andrea, C. (2000) Characterization and expression of DNA sequences encoding putative type-II metallothioneins in the seagrass 
Posidonia oceanica. Plant Physiol. 123, 1571-1581.

Thompson, J. D., Gibson, T. J., Plewniak, F., Jeanmougin, F. and Higgins, D. G. (1997) The ClustalX windows interface: flexible strategies for multiple sequence alignment aided by quality analysis tools. Nucleic Acids Res. 24, 4876-4882.

Ulmasov, T., Hagen, G. and Guilfoyle, T. J. (1999) Dimerization and DNA binding of auxin response factors. Plant J. 19, 309319.

Vallee, B. L. (1991) Introduction to metallothionein. Methods Enzymol. 205, 3-7.

van der Meer, I. M., Brouwer, M., Spelt, C. E., Mol, J. N. M. and Stuitje, A. R. (1992) The TACPyAT repeats in the chalcone synthase promoter of Petunia hybrida act as a dominant negative cis-acting module in the control of organ-specific expression. Plant J. 2, 525-535.

Vasak, M. and Hasler, D. W. (2000) Metallothioneins: new functional and structural insights. Curr. Opin. Chem. Biol. 4, 177-183.

Whitelaw, C. A., Le Huquet, J. A., Thurman, D. A. and Tomsett, A. B. (1997) The isolation and characterization of type II metallothionein-like genes from tomato (Lycopersicon esculentum L.). Plant Mol. Biol. 33, 503-511.
Wong, H. L., Sakamoto, T., Kawasaki, T., Umemura, K. and Shimamoto, K. (2004) Down-regulation of metallothionein, a reactive oxygen scavenger, by the small GTPase OsRac1 in rice. Plant Physiol. 135, 1447-1456.

Xu, N., Hagen, G. and Guilfoyle, T. (1997) Multiple auxin response modules in the soybean SAUR 15A promoter. Plant Sci. 126, 193-201.

Xu, Y., Yu, H. and Hall, T. C. (1995) Rice triosephosphate isomerase gene 5 'sequence directs beta-glucuronidase activity in transgenic tobacco but requires an intron for expression in rice. Plant Physiol. 106, 459-467.

Yu, L. H., Umeda, M., Liu, J. Y., Zhao, N. M. and Uchimiya, H. (1998) A novel MT gene of rice plants is strongly expressed in the node portion of the stem. Gene 206, 29-35.

Zhou, G. K., Xu, Y. F. and Liu, J. Y. (2005) Characterization of a rice class II metallothionein gene: tissue expression patterns and induction in response to abiotic factors. J. Plant Physiol. 162, 686-696.

Zhou, J. and Goldsbrough, P. B. (1995) Structure, organization and expression of the metallothionein gene family in Arabidopsis. Mol. Gen. Genet. 248, 318-328. 